\title{
SemanticFusion: Joint Labeling, Tracking and Mapping
}

\author{
Tommaso Cavallari $\left.{ }^{(}\right)$and Luigi Di Stefano \\ Department of Computer Science and Engineering, \\ University of Bologna, Bologna, Italy \\ \{tommaso.cavallari, luigi.distefano\}@unibo.it
}

\begin{abstract}
Kick-started by deployment of the well-known KinectFusion, recent research on the task of RGBD-based dense volume reconstruction has focused on improving different shortcomings of the original algorithm. In this paper we tackle two of them: drift in the camera trajectory caused by the accumulation of small per-frame tracking errors and lack of semantic information within the output of the algorithm. Accordingly, we present an extended KinectFusion pipeline which takes into account per-pixel semantic labels gathered from the input frames. By such clues, we extend the memory structure holding the reconstructed environment so to store per-voxel information on the kinds of object likely to appear in each spatial location. We then take such information into account during the camera localization step to increase the accuracy in the estimated camera trajectory. Thus, we realize a SemanticFusion loop whereby perframe labels help better track the camera and successful tracking enables to consolidate instantaneous semantic observations into a coherent volumetric map.
\end{abstract}

Keywords: SLAM - Deep learning $\cdot$ Semantic segmentation $\cdot$ Semantic fusion - Semantic camera tracking

\section{Introduction}

Since the publication of the KinectFusion paper by Newcombe and colleagues [1], significant interest has spurred on the topic of dense surface mapping and tracking by means of a handheld RGB-D sensor, this resulting in a multitude of proposals focused on extending and/or improving the original algorithm from a variety of diverse perspectives. Thus, for example, attention has been devoted to extend KinectFusion to reconstruct either large scale [2] or non-rigid [3] scenes, to improve the tracking module [4] or also to achieve detection of known object instances [5].

Electronic supplementary material The online version of this chapter (doi:10. 1007/978-3-319-49409-8_55) contains supplementary material, which is available to authorized users. 

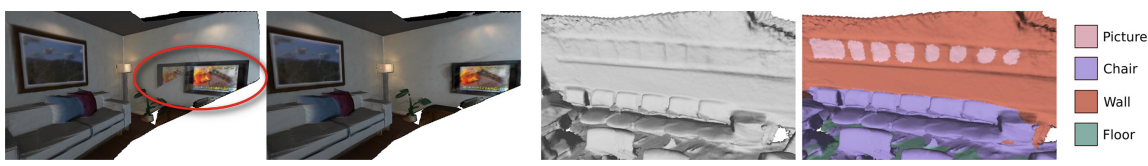

Fig. 1. Advantages of the proposed technique. Left: scene reconstruction provided by the geometry-based KinectFusion tracker shows gross errors due to the flat wall, while deployment of semantic cues enables correct handling of planar surfaces. Right: while KinectFusion is concerned with surface information only, our method can provide a semantically labeled volumetric reconstruction of the workspace.

Differently, the work described in this paper is concerned with endowing KinectFusion with the ability to create a semantically labeled dense reconstruction of the environment as well as with deploying per-frame semantic information to improve camera tracking, the two processes carried out jointly and synergistically. Indeed, on one hand we track the camera by relying also on per-pixel semantic labeling of the current RGB-D frame, so that a likely camera pose should explain not only the geometry of the scene but also its semantics: e.g., a pixel labeled as picture should preferably project onto a voxel tagged as picture than as wall. On the other hand, upon successful camera tracking we fuse the category map associated with the current frame into a volumetric representation, so that the final labeling results from suitable integration over time of the many fragile instantaneous observations delivered by the per-frame labeler. Thereby, semantic labeling ameliorates camera tracking, especially while acquiring scene elements - such as walls or windows - that may not provide distinctive geometric cues. Camera tracking then robustifies semantic labeling by allowing for identification of observations dealing with the same surface patch at different times so to assess their likelihood and confidence. The two key advantages brought in by our proposal are exemplified in Fig. 1.

Previous work has demonstrated how deployment of additional cues, such as color [6] or contours [4], can significantly improve the accuracy of the original KinectFusion tracker, which relies on geometrical information only. Unlike previous work, in this paper we advocate reliance on higher-level observations, such as per-pixel object category tags, to improve the tracking module and present a method that can also output automatically a fully labeled dense reconstruction of the environment with confidence scores for multiple object categories stored into each voxel of the mapped space. This kind of semantically rich output may vastly facilitate difficult tasks related to 3D indoor scene understanding, object discovery, object recognition and grasping, path planning and human/robot navigation.

\section{Related Work}

Newcombe's work [1], well-favored in its simplicity and effective in its core task - mapping of small/medium size environments rich in geometric structure suffers from the four main shortcomings highlighted as follows. (a) Reliance on 
a memory-demanding data structure located on the GPU, such as a dense fixedsize voxel grid, makes it impossible to map large scale scenes. (b) The drift error inherently accumulated during the tracking process may cause gross reconstruction errors when observing smooth/flat surfaces or closing camera path loops. (c) The requirement for static and rigid scenes hampers usability outside controlled settings, which severely limits the breadth of practical applications. (d) Finally, KinectFusion outputs a purely geometric map of the environment, thus providing no semantic hints to support higher level reasoning about sensor surroundings.

In the remainder of this section we will mention some works tackling each of the aforementioned issues and describe how our proposed technique addresses two of them originally.

(a) Different approaches can be taken to extend the mapped workspace: the reference frame for the volume can be moved alongside with sensor movements, possibly downloading parts of the voxel grid from GPU to CPU memory [7,8]; alternatively, sparse voxel grids indexed as hierarchical structures or Hash tables can be stored in memory to diminish occupancy and increase the bounds of the mappable space $[2,9]$.

(b) Some works have proposed to rely on additional clues with respect to the purely-geometric KinectFusion tracker to attempt reducing the inherent drift error. In particular, Bylow and Olsson [6] show that injecting perpixel color measurements within the cost function optimized by the tracker does improve accuracy, especially when dealing with flat or smooth surfaces that present distinctive color features. Conversely, Zhou and Koltun [4] posit to deploy occluding contours rather than color and demonstrate the effectiveness of their proposal in experiments focused on scanning individual objects featuring smooth and evenly colored surfaces. Other approaches concern tackling drift by a global optimization step on a pose graph, which may better counteract misalignments showing up at loop closures. The pose optimization step may be run either off-line, after the capture process is terminated, or on-line, by continuously optimizing the poses of partial volumes, possibly deforming the surfaces contained therein [10-13].

(c) Lightly dynamic scenes are allowed by the original KinectFusion algorithm, though only due to the volume update step behaving as a low-pass filter that forgets old measurements after a certain number of frames. However, scene motion is not explicitly considered, sudden movements of relatively large-size objects inevitably causing tracking failures. Recent works, instead, have demonstrated impressive results in non-rigid and dynamic settings by modeling motion as a fundamental property of the captured scene $[3,14]$.

(d) The work by Fioraio et al. [5] attempts to gather semantic knowledge while tracking and mapping by KinectFusion. In particular, deployment of their Semantic Bundle Adjustment framework [15] together with KinectFusion enables to detect known object instances, estimate their 6 DOF poses and embed objects into a globally optimized pose graph which helps counteracting drift. Although pursuing a diverse graph-based mapping strategy, the SLAM++ system by Salas-Moreno et al. [16] relies on the detection of known 
object instances to perform camera tracking by an approach akin to KinectFusion. On the other hand, Valentin et al. [17] show compelling results in the task of interactively labeling a scene already reconstructed via KinectFusion. Their approach combines user interaction with on-line learning by streaming random forests to obtain per-voxel semantic labels representing personalized categories of the objects present in the environment. Eventually, the very recent paper by Cavallari and Di Stefano [18] describes the first attempt to obtain automatically a labeled volumetric reconstruction. Here, the authors track the camera by a standard geometric KinectFusion approach and, upon successful tracking, fuse the labels delivered by a perframe semantic segmentation algorithm into the voxel grid by means of a simple evidence counting scheme that allows storing information concerning a single category per voxel only.

Therefore, the first contribution provided by this paper deals with the issue of the inherent drift that may be accumulated by a purely geometric tracker. Unlike previous work, we propose to rely on semantic observations such as category labels rather than low-level cues like color [6] or occluding contours [4]. The second contribution concerns gathering not only structure but also semantics, our proposal delivering a densely labeled volumetric reconstruction of the workspace. While previous work [17] has addressed interactive scenarios and shown how to obtain a similar output by placing the user in the loop, in this paper we propose a fully automatic approach. With respect to the automatic labeling method recently reported in [18], our proposal is not constrained to keeping track of only one label per voxel but instead can gather evidence concerning all categories across the whole voxel grid. As it will be discussed in Subsect. 3.2, besides providing a semantically richer output, mantaining information about all categories in each voxel is instrumental to the realization of a semantic tracker. Moreover, while the works in $[17,18]$ assume surface reconstruction to be achieved by standard KinecFusion, we realize a closed loop between the semantic labeling and the dense mapping and tracking processes, so to allow one process to beneficially influence the other. In this respect, our approach is more similar in spirit to previous works aimed at creating synergistic interactions between object detection and SLAM $[5,15,16]$.

\section{Description of the Method}

A TSDF (Truncated Signed Distance Function) volume employed during the execution of a KinectFusion-like tracking and mapping pipeline typically contains the following information in each voxel:

1. Truncated signed distance of the voxel from the closest world surface.

2. Weight used to compute the running average during the voxel update step.

3. (Optional) RGB values representing the color of the surface and associated weights. 
To obtain a semantically labeled voxel, we store also the category labels as a histogram wherein the value stored in each bin is as higher as the corresponding label is observed more frequently and with higher confidence scores. As such, a bin value becomes correlated to the probability that an object of a certain category is located at the voxel's $3 \mathrm{D}$ position. This representation allows us to both compare the evidence about the diverse categories gathered within each voxel as well as to rank the labeling confidence between different voxels.

Without loss of generality, we assume to employ a semantic labeling algorithm capable to output a per-pixel category label for each input frame together with an associated score map representing the degree of belief in the predicted pixel labels. Indeed, this is the most typical kind of output delivered by semantic segmentation algorithms working with either RGB or RGB-D images. Should the labeler at hand provide a more comprehensive output, such as a full pmf across categories at each pixel position, we would easily remap it into the format currently accepted by our method. In the following subsections we will describe in detail how to augment the standard KinectFusion volume update technique to account also for per-pixel labels and how to deploy such semantic information to robustify the tracking process.

\subsection{Volume Update Process}

The update process allows for integration of a new measurement into the volume capturing the structure of the world as previously seen by the sensor. We denote with $T=(R, \mathbf{t}) \in \mathbb{S E}_{3}$ the affine transformation mapping points in the camera reference frame to world coordinates and $\mathbf{u}=(u, v)^{T}$ the coordinates of a pixel in the input frame. We also assume the intrinsic parameters of the camera $\left(f_{x}, f_{y}, c_{x}, c_{y}\right)$ to be known, so to be able to perform perspective projection of $3 \mathrm{D}$ points into pixels and vice-versa. We will indicate the projection operation as $\pi(\mathbf{p}): \mathbb{R}^{3} \rightarrow \mathbb{R}^{2}$ and reprojection as $\pi^{-1}(\mathbf{u}, z):\left(\mathbb{R}^{2}, \mathbb{R}\right) \rightarrow \mathbb{R}^{3}$. Thus, as soon as a new input frame has been tracked, we wish to integrate into the volume the depth image, $d(\mathbf{u})$, the RGB image, $i(\mathbf{u})$, as well as the pair of maps, $l(\mathbf{u})$ and $s(\mathbf{u})$, representing, respectively, the per-pixel labels and scores yielded by the semantic labeler.

The original KinectFusion algorithm [1] updates the volume by visiting each voxel and projecting the $3 \mathrm{D}$ location of its center, $\mathbf{p}_{\mathbf{v}}$, onto the image plane according to the tracked pose and the projection function:

$$
\mathbf{u}_{\mathbf{v}}=\pi\left(T^{-1} \mathbf{p}_{\mathbf{v}}\right)
$$

Then, if the projected location falls within the bounds of the input image, the data contained in the voxel get updated. The signed distance from the voxel center to the surface described by the depth image is computed by considering the $z$ coordinate of the voxel center in the camera reference frame:

$$
S D F_{v}=\left(T^{-1} \mathbf{p}_{\mathbf{v}}\right)_{z}-d\left(\mathbf{u}_{\mathbf{v}}\right)
$$


A truncation distance, $\delta$, is then considered, so that only voxels within the sensor view frustum and whose $S D F_{v} \geq-\delta$ are updated. The voxel TSDF value, $D$, and associated weight, $W_{d}$, are modified as follows:

$$
\begin{aligned}
T S D F_{v} & =\left\{\begin{array}{c}
1 \text { for } S D F_{v} \geq \delta \\
\frac{S D F_{v}}{\delta} \text { for }-\delta \leq S D F_{v} \leq \delta
\end{array}\right. \\
D^{t+1}\left(\mathbf{p}_{\mathbf{v}}\right) & =\frac{D^{t}\left(\mathbf{p}_{\mathbf{v}}\right) W_{d}^{t}\left(\mathbf{p}_{\mathbf{v}}\right)+T S D F_{v}}{W_{d}^{t}\left(\mathbf{p}_{\mathbf{v}}\right)+1} \\
W_{d}^{t+1}\left(\mathbf{p}_{\mathbf{v}}\right) & =\min \left(W_{d}^{t}\left(\mathbf{p}_{\mathbf{v}}\right)+1, \text { MaxWeight }\right)
\end{aligned}
$$

with superscripts $t$ and $t+1$ denoting the current and updated values, respectively. Thus, the quantity stored in a voxel amounts to a running average over time of the measured TSDF values. The saturation of the weight (Eq. 5) allows for forgetting old values, which, in turn, enables mapping of slightly dynamic scenes. Other weighting schemes aimed at possibly improving the volume update process have been shown in literature $[1,6,19]$. Then, as for integration of the color data acquired from the RGB image, $i(\mathbf{u})$, we follow the approach presented in the paper by Bylow and colleagues [6].

In principle, updating the bins of the label histogram associated with a voxel may be performed similarly to the just described TSDF and color updating processes. However, as already mentioned, we assume the semantic segmentation algorithm to provide a single label and associated confidence score at each pixel position of the input frame. When integrating a new frame then, at each voxel we would be able to update only the bin associated with the label observed at the corresponding pixel and we are faced with the issue of whether and how to update the other bins. Updating only the observed bin would inevitably result in the plateauing of the histogram at relatively high values in case several highly confidently and incoherent labels get integrated into a voxel. Instead, we devised a suitable updating technique that tackles this issue by both increasing the value of the bin associated with the observed label as well as decreasing those of all other bins. In particular, the increment is proportional to the confidence score provided by the labeler while the decrement factor takes also into account the evidence hitherto gathered into the histogram to penalize less higher bins.

Formally, denoting the category histogram stored in each voxel as $L \in \mathbb{R}^{N}$, with $N$ the number of categories of interest, we define the weight update factor for each label $i$ based on the label assigned to the pixel, $l(\mathbf{u})$, and the corresponding score $s(\mathbf{u})$ :

$$
w_{l_{i}}=\left\{\begin{array}{r}
s(\mathbf{u}) \text { for } i=l(\mathbf{u}) \\
L_{i}^{t}\left(\mathbf{p}_{\mathbf{v}}\right)(1-s(\mathbf{u})) \text { for } i \neq l(\mathbf{u})
\end{array}\right.
$$

Then, we update each histogram bin as follows:

$$
L_{i}^{t+1}\left(\mathbf{p}_{\mathbf{v}}\right)=\frac{L_{i}^{t}\left(\mathbf{p}_{\mathbf{v}}\right) W_{d}^{t}\left(\mathbf{p}_{\mathbf{v}}\right)+w_{l_{i}}}{W_{d}^{t}\left(\mathbf{p}_{\mathbf{v}}\right)+1}
$$


It worth observing that, analogously to TSDF and color, the label update step is tantamount to a running average over the number of observations for a voxel.

\subsection{Tracking Algorithm}

To update the information stored into the volume by integrating new measurements, we need to track the RGB-D sensor as it moves within the environment. In KinectFusion [1] camera tracking is performed by ICP-based alignment between the surface associated with the current depth image and that extracted from the TSDF. Later, Bylow et al. [19] and Canelhas et al. [20] proposed to track the camera by direct alignment of the current depth image to the mapped environment encoded into the TSDF as the zero-level isosurface. This approach has been proven to be faster and more accurate than the original KinectFusion tracker.

Camera tracking by direct alignment of the acquired depth image with respect to the TSDF volume relies on the following consideration: assuming that the estimated pose of the camera is correct and noise does not affect the current and previous depth measurements, then each depth pixel should correspond to the projection of a 3D world point that, given the content of the TSDF grid, features a null distance function (i.e. lies on the surface). Clearly, noiseless depth images and perfectly accurate camera pose estimations cannot be obtained in real settings. However, Bylow et al. [19] show that for small camera movements (as it is the case during real-time tracking) iteratively minimizing the sum of the squared distances between the 3D points corresponding to depth pixels and their corresponding TSDF values allows for accurate estimation of camera poses.

In this paper, we propose to consider also the category labels assigned to pixels within the objective function minimized by the tracker. Indeed, a shortcoming of both the standard KinectFusion ICP tracking algorithm and the tracker based on direct alignment of depth images consists in the difficulty to localize the camera when the acquired frames depict scenes poor in geometry such as smooth or flat surfaces. We argue that exploiting the inherent structure determined by the boundaries between labeled objects can improve tracking accuracy when the scene features insufficient geometric clues.

Camera pose estimation is thus performed by finding the affine transformation, $T$, that minimizes a cost function consisting of two separate terms. One term measures the geometric alignment of the depth frame to the surface implicitly encoded into the TSDF, while the other term captures the coherence between the per-pixel labeling proposed by the semantic segmentation algorithm with respect to the label histograms already stored into voxel grid. We define $\mathbf{p}_{\mathbf{u}}$ as the $3 \mathrm{D}$ location of the point determined by a pixel $\mathbf{u}$ having a valid value in the depth image:

$$
\mathbf{p}_{\mathbf{u}}=\pi^{-1}(\mathbf{u}, d(\mathbf{u}))
$$

The geometric error term is then given by the truncated signed distance from a voxel to the closest surface:

$$
E_{d}(T, \mathbf{u})=D\left(T \mathbf{p}_{\mathbf{u}}\right)
$$


We define the semantic error term based on both the confidence of the labeler in the predicted pixel label and the amount of evidence already gathered on that label within the corresponding voxel:

$$
E_{l}(T, \mathbf{u})=s(\mathbf{u})\left(1-L_{l(\mathbf{u})}\left(T \mathbf{p}_{\mathbf{u}}\right)\right)
$$

Thereby, the error term turns out high when the per-pixel labeler confidently predicts a category for which the degree of previous evidence is small.

The final objective function to be minimized by the tracker takes the following form:

$$
E(T)=\sum_{\mathbf{u}}\left(E_{d}(T, \mathbf{u})^{2}+\alpha E_{l}(T, \mathbf{u})^{2}\right)
$$

Given a candidate pose, the first term of the sum quantifies the geometric misalignment between the current frame and the surface embedded into the TSDF. The second, instead, penalizes those poses where the labels assigned to pixels turn out incoherent with respect to the category histograms stored into the corresponding voxels. As such, the second term of the sum may be thought of as quantifying the semantic misalignment between the current frame and the volumetric map. The blending parameter, $\alpha$, enables to weigh properly the contribution of the two error terms.

By employing a minimal parametrization $\xi=\left(v_{x}, v_{y}, v_{z}, \omega_{x}, \omega_{y}, \omega_{z}\right) \in \mathbb{R}^{6}$ of the $T=(R, \mathbf{t}) \in \mathbb{S} \mathbb{E}(3)$ transformation, and knowing that the Lie algebra allows expressing $T=e^{\xi}$ and $\xi=\ln (T)$ via the exponential and logarithm map operations [21], we can define:

$$
E_{d \mathbf{u}}(\xi)=D\left(e^{\xi} \mathbf{p}_{\mathbf{u}}\right)
$$

and

$$
E_{l \mathbf{u}}(\xi)=s_{\mathbf{u}}\left(1-L_{l \mathbf{u}}\left(e^{\xi} \mathbf{p}_{\mathbf{u}}\right)\right)
$$

so to rewrite Eq. 11 as:

$$
E(\xi)=\sum_{\mathbf{u}}\left(E_{d \mathbf{u}}(\xi)^{2}+\alpha E_{l \mathbf{u}}(\xi)^{2}\right)
$$

Using Eq. 14 we can finally express the camera tracking cost function as:

$$
\xi=\underset{\xi}{\operatorname{argmin}} \sum_{\mathbf{u}}\left(E_{d \mathbf{u}}(\xi)^{2}+\alpha E_{l \mathbf{u}}(\xi)^{2}\right)
$$

By linearizing the function around an initial pose $\hat{\xi}$ (such as the estimated camera pose for the previously tracked frame) and assuming a small movement between the two acquisitions (as mentioned before, a reasonable assumption in the hand-held tracking scenario), it is possible to perform an iterative nonlinear minimization using a method such as Levemberg-Marquardt in order to estimate the camera pose, $\xi$, that optimally aligns the current frame to the current volumetric reconstruction both geometrically and semantically. 
The gradient of the cost function required by the iterative minimization to compute the increment applied to $\xi$ is attained independently for the two terms. As for the gradient of the geometric term, in each pixel $\mathbf{u}$, we trilinearly interpolate the values of the TSDF in the 8 voxels closest to point $e^{\xi} \mathbf{p}_{\mathbf{u}}$. The gradient for the semantic error term is also computed by trilinear interpolation, though, in this case, of the values $1-L_{l(\mathbf{u})}(\mathbf{x})$, with $\mathbf{x}$ representing voxel coordinates. Thereby, for each pixel we consider the category histogram bin associated to the label assigned to the pixel itself. It is worthwhile observing that optimization of the semantic error mandates maintaining in each voxel an histogram concerning the likeliness of all categories, as we propose in this paper, whilst storing the evidence gathered for the most likely label only, as described in [18], would make it impossible to compute the gradient of the semantic error due to voxels lacking the information on whether moving along a certain direction would either increase of decrease the likeliness of the sensed pixel label.

\section{Experimental Evaluation}

In this section we present quantitative (Subsect. 4.1) and qualitative (Subsect. 4.2) results showing how the proposed camera localization algorithm based on both geometry and semantics can successfully reduce the trajectory error in several challenging sequences. Purposely, we employ the well-known RGB-D SLAM Dataset by Sturm et al. [22] and the ICL NUIM Dataset by Handa and colleagues [23]. We also show reconstructed environments obtained from sequences part of the Sun3D Dataset [24] together with a sequence depicting a household environment.

As mentioned in Sect. 3, to successfully track the camera pose and update the volume, we assume to be provided in each frame with per-pixel labels and confidence scores by a semantic segmentation algorithm. In our tests, we employed the Fully Convolutional Networks recently proposed by Long and colleagues [25]. Such technique, based on a Convolutional Neural Network, performs an image labeling suitable to our needs by computing, for each pixel in the input image, a complete probability distribution over all the categories of interest. We then select the label having the maximum probability and use such values to populate the $l(\mathbf{u})$ and $s(\mathbf{u})$ matrices given as input to our algorithm. The authors provide several pretrained networks ${ }^{1}$ based on different sets of object categories and input features. We considered two such networks, one based on the 59 categories of the Pascal Context dataset [26] (FCN-8s PASCAL-Context, as the authors name it in their paper) and another trained on 40 categories of the NYUDv2 dataset [27] (FCN-16s NYUDv2). While the FCN-8s network process only the input RGB frame to obtain the categories, the network trained on the NYUD dataset requires also computation of the HHA depth embedding [28]. The deployment of either network requires a vast amount of GPU computing power and memory but the latter, relying on processing both an RGB and HHA image,

\footnotetext{
${ }^{1}$ https://github.com/BVLC/caffe/wiki/Model-Zoo\#fcn.
} 
needs twice the GPU RAM as the former (about $8.8 \mathrm{~GB}$ ), which reduces significantly the GPU memory available to store the proposed voxel grid containing category histograms. Eventually we decided to privilege the FCN-8s PASCALContext network in our experimental evaluation. We point out, though, that our proposed semantic tracking technique does not rely on a specific image labeling algorithm but merely expects to be provided with per-pixel labels and scores.

As between the 59 categories detected by the aforementioned labeling algorithm we are interested only in a subset comprising indoor objects (e.g. "tables", "chairs", "walls", "tv monitors" and not categories such as "sheep" or "mountain"), we filter the network output at each pixel so to keep the most likely label within the subset of categories of interest. All the performed tests consider 12 categories. Each voxel has the following layout in memory:

TSDF Value Half precision float, 2 bytes

TSDF Weight Half precision float, 2 bytes

Color R,G,B and weight channels stored each as unsigned char, 4 bytes

Category histogram Each bin as a floating point value in the interval [0..1], mapped as [0..255] into an unsigned char, 12 bytes

The total occupancy for a $512^{3}$ voxel grid is therefore $\sim 2.5$ GB of GPU memory, well within the possibilities of modern graphic cards.

We would like to point out that, with a dense volume-based mapping approach, the memory footprint of the system exhibits a cubic dependence on both the voxel and workspace sizes. Comparatively, increasing the amount of bytes stored in each voxel increases memory occupancy only linearly. Thus, storing 12 more bytes in each voxel, as we do for labels, does not change the working constraints of the system dramatically. To clarify this claim, let us assume the extent of the environment to be $8^{3} \mathrm{~m}^{3}$ : by employing a quite standard $512^{3}$ voxel grid we would be able to map the environment with a resolution of $\sim 1.6 \mathrm{~cm}$, this requiring $1 \mathrm{~GB}$ of GPU memory without storing per voxel-labels and, as mentioned before, $\sim 2.5 \mathrm{~GB}$ when also storing an histogram with 12 label scores in each voxel. Conversely, should we wish to map the same environment with a voxel resolution of $0.5 \mathrm{~cm}$, a grid of $1600^{3}$ voxels would be necessary, this requiring $32 \mathrm{~GB}$ of GPU memory without per voxel-labels and $\sim 80 \mathrm{~GB}$ in case of the semantic voxel grid. Both cases are intractable with the current graphics hardware thus, in practice, with the proposed system we are able to handle workspaces of similar size as KinectFusion.

\subsection{Quantitative Results}

As mentioned, we evaluate the performances of the proposed label-aware tracking method on RGB-D sequences part of the TUM [22] and ICL_NUIM [23] datasets. As customary in the evaluation of SLAM algorithms, we deploy the RMS Absolute Trajectory Error performance metric described by Sturm et al. [22]. We compare our approach to a standard geometry-based tracker based on direct alignment of the depth image as well as to the improved tracker that employs 
also color information [6]. As the article describing the color-based tracker did not consider the ICL_NUIM dataset in the evaluation, we implemented their tracking algorithm and show here the RMS ATE also for our implementation.

As for the runtime configuration of our algorithm, we employ a voxel grid spanning $8^{3} \mathrm{~m}^{3}$ of space. The truncation distance has been set to $0.3 \mathrm{~m}$ and the $\alpha$ blending coefficient applied to our semantic error term to 0.085 , after a grid search over the parameter space.

Figure 2 shows the accuracy of different tracking approaches in several sequences of the RGBD-SLAM Dataset. Overall, we observe that most TUM sequences can be tracked quite successfully by a purely geometric approach, so that both semantics and color have got no chances to bring in notable improvements in accuracy. Nor they cause any harm, though. Instead, in those sequences, like "floor" and "room", turning more challenging for a purely geometric tracker, employment of additional clues, such as semantic labels - or color - does help reducing the tracking error quite significantly. Indeed, the improvement achievable by our semantic tracker versus a purely geometric approach is much higher in "room" than in "floor", as the former sequence is characterized by a richer semantic content (i.e. presence of several object categories) while in the latter the sensor continuously observes the floor of a room, which renders color cues more distinctive than semantics.

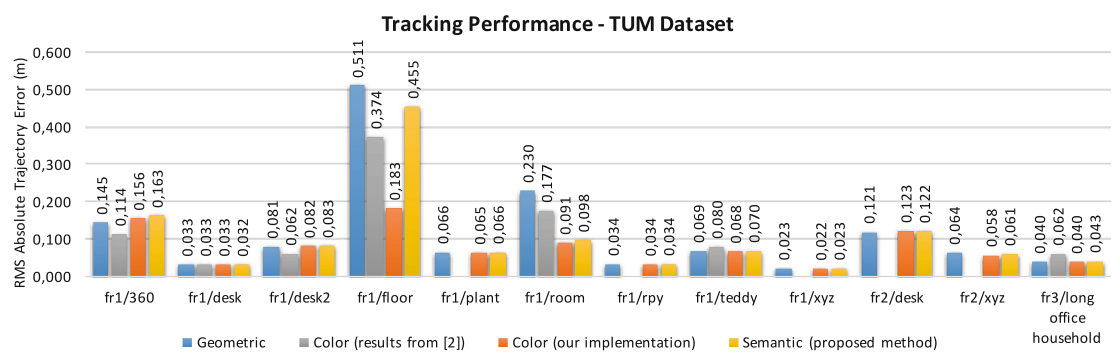

Fig. 2. Tracking performances in sequences part of the TUM [22] dataset. Geometric represents the purely geometric KinectFusion tracker. For the Color-based approach we show both the results reported by Bylow in [6] $(\alpha=0.1)$, as well as those achieved by our own implementation of Bylow's approach. Semantic denotes our proposal.

Figure 3 shows the tracking error on the sequences of the ICL_NUIM dataset. Handa et al. [23] provide two sets of four RGBD sequences obtained by rendering views from a synthetically generated model of two environments: a living room and an office. One set contains noiseless images while the depth frames in the other have been corrupted by noise akin to that present in the images acquired by a real Kinect sensor. In our tests we consider the latter, noisy, set. Once again, it can be observed how, in those sequences where the geometric tracker has more difficulties in estimating the correct camera trajectory, deployment of semantic labels can ameliorate tracking accuracy notably. Moreover, unlike 


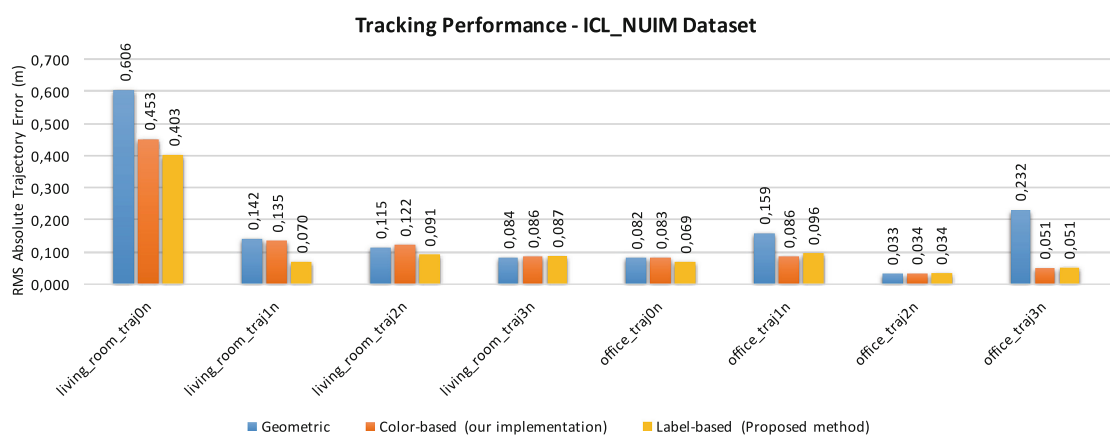

Fig. 3. Tracking performances in sequences part of the ICL_NUIM [23] dataset. Series as in Fig. 2.

the TUM dataset, it turns out here that, more often than not, semantic labels compare favorably with respect to color cues, possibly due to the richer semantic content of the scenes.

Finally, Zhou and Koltun [4] evaluate their contour-based tracking method on a small subset of the TUM dataset, i.e. four sequences, each focused on scanning a single object featuring smooth and evenly colored surfaces. In Fig. 4 we report the trajectory errors for the considered tracking methods, including Zhou's, on the four sequences used for the evaluation in [4]. Clearly, scanning a single object is neither the typical operating mode nor particularly suitable a scenario to our method, which, instead, is aimed at automatic reconstruction and volumetric labeling of relatively large workspaces featuring a number of diverse categories of interest (indeed, we set the volume size to $2.5^{3} \mathrm{~m}^{3}$ in these four sequences).

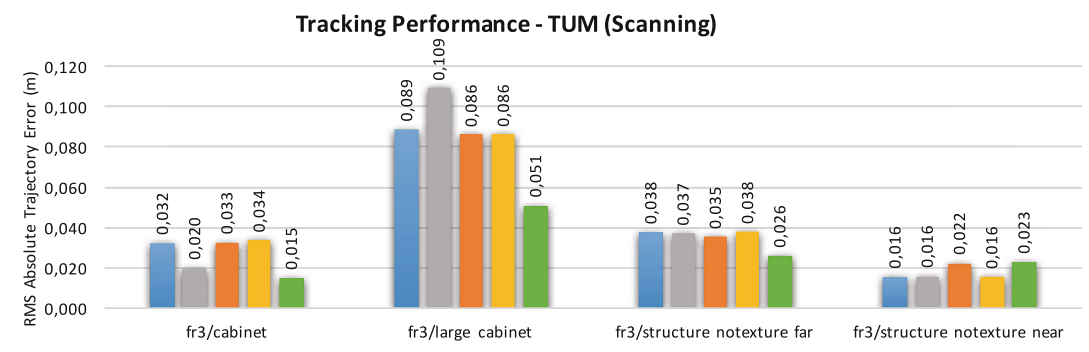

= Geometric $=$ Color (results from [4]) =Color (our implementation) $=$ Semantic (proposed method) $\square$ Contour (results from [4])

Fig. 4. Tracking performances in the object scanning sequences considered in [4]. The method proposed in [4] is referred to as Contour. As for the original color based tracker, we show here the results reported in [4], which were obtained by the authors running Bylow's code. As usual, we also provide the results attained by our own implementation of Bylow's tracker. 
Yet, Fig. 4 vouches how employing semantic labels in object scanning settings is not detrimental to the overall tracking accuracy. Contours seem the most effective cues in these settings, while the results yielded by semantic labels may be judged on par to those attainable by deploying colors.

\subsection{Qualitative Results}

In this section we show, at first, qualitative results proving the advantages attainable by employing a label-aware camera tracker within a KinectFusion framework.

Figure 5 concerns an RGB-D sequence that we captured via a Kinect sensor in a corridor within a household environment. While the flatness of the wall prevents successful tracking based solely on the geometry of the surfaces, the low confidence of the "wall" label associated with the paintings provides enough distinctive cues to enable correct reconstruction of the environment.
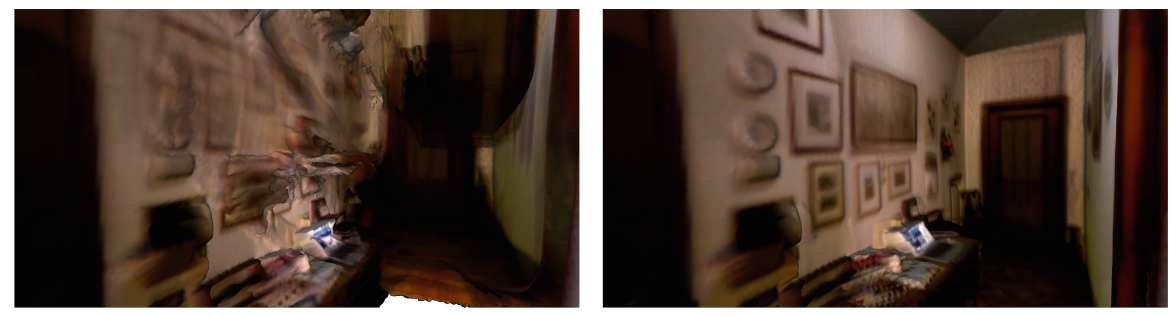

Fig. 5. Household scene captured with a kinect sensor. Left: a purely geometric tracker cannot estimate camera poses accurately due to the flatness of the wall. Right: deployment of semantic labels enable to map the environment correctly.

Figure 6 deals with the hotel room sequence contained in the SUN3D dataset [24]. When mapping the hotel's bathroom, the camera briefly lingers on the mirror above the faucet. An RGBD sensor such as the Kinect is unable to detect the mirror as a flat surface, instead mirroring the shape of the reflected environment as a window on a different room. A purely geometric tracking, thus, is unable to estimate the correct camera trajectory, resulting in a very bad reconstruction such as that shown by the left picture. However, the correct and coherent semantic labels associated to the surfaces surrounding the mirror deployed in our tracking cost function allow for better constraining camera poses and obtain an accurate reconstruction. The mirror still appears as a hole in the wall showing the rest of the hotel room because depth measurements in that spatial location are farther away than the mirror itself, but the rest of the bathroom is correctly reconstructed.

Finally, we provide qualitative results to demonstrate the capability of our method to output semantically labeled volumes. Additional results are available in the supplementary material. Figure 7 shows details of the "room" sequence 

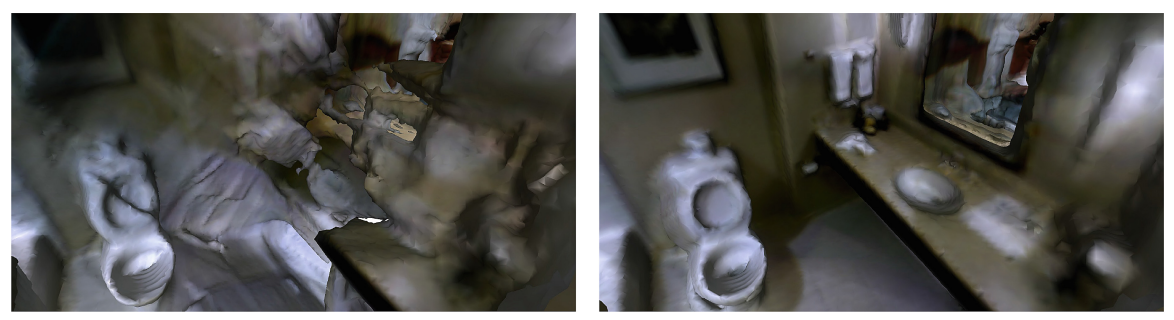

Fig. 6. Fatal tracking failure prevented. Left: the mirror causes a purely geometric approach to fail, the reconstructed volume turning out unrecognizable. Right: tracking succeeds and the bathroom is correctly mapped thanks to semantic labels.

(TUM dataset, top row) and the aforementioned household sequence (also depicted in Fig. 5, bottom row), with each voxel labeled according to the tag exhibiting the highest score in the category histogram and the corresponding confidence shown as a heat map. It can be observed that most voxels are labeled correctly and confidence heat maps are quite reliable, due to high confidence labels unlikely turning out wrong and mislabeled areas featuring low scores. Moreover, the maps provide evidence on the presence of both large scene structures as well as smaller objects. It is worth highlighting that with the "room" sequence the quality of the $3 \mathrm{D}$ reconstruction is equivalent to that achievable by deploying color cues (see Fig. 2) however, by our method, one can also gather high level information concerning which types of objects are present in the environment and where they are located in space.
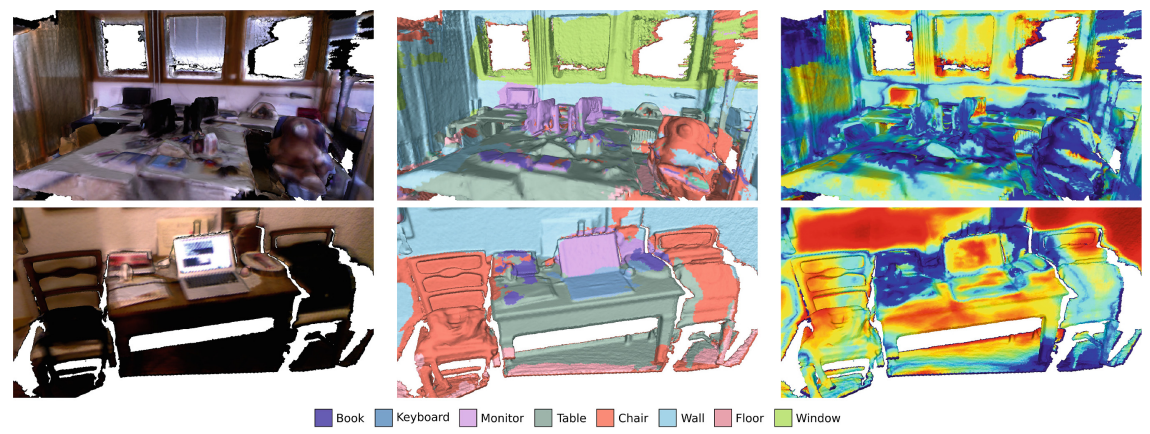

Fig. 7. Details of the reconstruction from the "room" sequence (TUM Dataset, Top) and household sequence (Fig. 5, Bottom). Left: RGB mesh. Center: labels associated to the reconstructed volume. Right: confidence scores assigned to the labels (blue: low - red: high). (Color figure online) 


\section{Concluding Remarks}

We have shown how high-level observations, such as per-pixel object category tags, can improve the dense mapping and tracking process popularized by KinectFusion when dealing with surfaces featuring scarce geometric cues. Quantitative experiments on standard benchmark datasets suggest that, overall, reliance on semantic labels for tracking yields results comparable to deployment of color. However, we believe that semantic information holds the potential to enable more reliable tracking as category tags are less affected by nuisances such as light changes, shadows, reflections, blur. For example, a labeler working only on depths would allow to semantically track the camera in very low-light conditions or even in the dark. More generally, we expect the performance of semantic tracking to improve alongside advances in labeling algorithms, our framework allowing for accommodating such foreseeable advances seamlessly. Also, peculiarly with respect to previous work aimed at robustifying the KinectFusion tracker, our proposal automatically outputs a semantically labeled volume wherein each voxel is endowed with the likeliness of the whole set of categories of interest. The semantic map attainable by our method seems to provide valuable cues to facilitate indoor scene understanding, as it would typically detect and coarsely localize major large-size scene structures such as floor, walls, tables, windows, chairs as well as several smaller objects like monitors, books, keyboards. Such information comes together with a reliable confidence map that may be deployed effectively within the high-level reasoning process.

The first major limitation of our approach concerns speed. Indeed, while the semantic tracker can run comfortably at about 50-60 fps, the Fully Convolutional Network we currently rely upon for semantic labeling takes about $250 \mathrm{~ms}$ per frame, which brings down the overall frame rate of our system at about 3-4 fps. Yet, as our proposal is agnostic to the actual labeler, we are investigating on alternatives that may possibly improve speed without overly penalizing accuracy. The second limitation deals with our current fixed-size volume approach, which hinders the possibility to semantically map large workspaces such as big rooms or even multiple rooms. To address this, we are currently integrating Voxel Hashing [2] within our framework.

Acknowledgements. We gratefully acknowledge the support of NVIDIA Corporation with the donation of the Tesla K40 GPU used for this research.

\section{References}

1. Newcombe, R.A., Davison, A.J., Izadi, S., Kohli, P., Hilliges, O., Shotton, J., Molyneaux, D., Hodges, S., Kim, D., Fitzgibbon, A.: KinectFusion: real-time dense surface mapping and tracking. In: 2011 10th IEEE International Symposium on Mixed and Augmented Reality, pp. 127-136. IEEE, October 2011

2. Nießner, M., Zollhöfer, M., Izadi, S., Stamminger, M.: Real-time 3D reconstruction at scale using voxel hashing. ACM Trans. Graph. 32(6), 1-11 (2013) 
3. Newcombe, R.A., Fox, D., Seitz, S.M.: DynamicFusion: reconstruction and tracking of non-rigid scenes in real-time. In: 2015 IEEE Conference on Computer Vision and Pattern Recognition (CVPR), pp. 343-352. IEEE, June 2015

4. Zhou, Q.-Y., Koltun, V.: Depth camera tracking with contour cues. In: 2015 IEEE Conference on Computer Vision and Pattern Recognition (CVPR), pp. 632-638. IEEE, June 2015

5. Fioraio, N., Cerri, G., Di Stefano, L.: Towards semantic kinectfusion. In: Petrosino, A. (ed.) ICIAP 2013. LNCS, vol. 8157, pp. 299-308. Springer, Heidelberg (2013). doi:10.1007/978-3-642-41184-7_31

6. Bylow, E., Olsson, C.: Robust camera tracking by combining color and depth measurements. In: 2014 22nd International Conference on Pattern Recognition (ICPR) (2014)

7. Roth, H., Marsette, V.: Moving volume kinectfusion. Proc. Br. Mach. Vis. Conf. 112(1-112), 11 (2012)

8. Whelan, T., Kaess, M., Fallon, M.: Kintinuous: spatially extended kinectfusion. In: Robotics Science and Systems (Workshop on RGB-D: Advanced Reasoning with Depth Cameras) (2012)

9. Chen, J., Bautembach, D., Izadi, S.: Scalable real-time volumetric surface reconstruction. ACM Trans. Graph. 32(4) (2013)

10. Henry, P., Fox, D., Bhowmik, A., Mongia, R.: Patch volumes: segmentation-based consistent mapping with RGB-D cameras. In: 2013 International Conference on 3D Vision, pp. 398-405 (2013)

11. Zhou, Q.Y., Miller, S., Koltun, V.: Elastic fragments for dense scene reconstruction. In: 2013 IEEE International Conference on Computer Vision, pp. 473-480. IEEE, December 2013

12. Zhou, Q.Y., Koltun, V.: Dense scene reconstruction with points of interest. ACM Trans. Graph. 32(4), 112:1-112:8 (2013)

13. Fioraio, N., Taylor, J., Fitzgibbon, A., Di Stefano, L., Izadi, S.: Large-scale and drift-free surface reconstruction using online subvolume registration. In: 2015 IEEE Conference on Computer Vision and Pattern Recognition (CVPR), pp. 4475-4483. IEEE, June 2015

14. Dou, M., Taylor, J., Fuchs, H., Fitzgibbon, A., Izadi, S.: 3D scanning deformable objects with a single RGBD sensor. In: 2015 IEEE Conference on Computer Vision and Pattern Recognition (CVPR), pp. 493-501. IEEE, June 2015

15. Fioraio, N., Di Stefano, L.: Joint detection, tracking and mapping by semantic bundle adjustment. In: Proceedings of the IEEE Computer Society Conference on Computer Vision and Pattern Recognition, pp. 1538-1545, June 2013

16. Salas-Moreno, R.F., Newcombe, R.A., Strasdat, H., Kelly, P.H., Davison, A.J.: SLAM++: simultaneous localisation and mapping at the level of objects. In: 2013 IEEE Conference on Computer Vision and Pattern Recognition, pp. 1352-1359, June 2013

17. Valentin, J., Vineet, V., Cheng, M.M., Kim, D., Shotton, J., Kohli, P., Niessner, M., Criminisi, A., Izadi, S., Torr, P.: SemanticPaint: interactive 3D labeling and learning at your fingertips. ACM Trans. Graph. (TOG) (2015)

18. Cavallari, T., Di Stefano, L.: Volume-based semantic labeling with signed distance functions. In: Pacific Rim Symposium on Image and Video Technology (2015)

19. Bylow, E., Sturm, J., Kerl, C., Kahl, F., Cremers, D.: Real-time camera tracking and 3D reconstruction using signed distance functions. In: Robotics: Science and Systems (RSS) (2013) 
20. Canelhas, D.R., Stoyanov, T., Lilienthal, A.J.: SDF Tracker: a parallel algorithm for on-line pose estimation and scene reconstruction from depth images. In: IEEE International Conference on Intelligent Robots and Systems, pp. 3671-3676 (2013)

21. Blanco, J.: A tutorial on SE(3) transformation parameterizations and on-manifold optimization. University of Malaga. Technical Report (3) (2010)

22. Sturm, J., Engelhard, N., Endres, F., Burgard, W., Cremers, D.: A benchmark for the evaluation of RGB-D SLAM systems. In: 2012 IEEE/RSJ International Conference on Intelligent Robots and Systems, pp. 573-580, October 2012

23. Handa, A., Whelan, T., McDonald, J., Davison, A.J.: A benchmark for RGBD visual odometry, 3D reconstruction and SLAM. In: 2014 IEEE International Conference on Robotics and Automation (ICRA), pp. 1524-1531, May 2014

24. Xiao, J., Owens, A., Torralba, A.: SUN3D: a database of big spaces reconstructed using SfM and object labels. In: Proceedings of the IEEE International Conference on Computer Vision, pp. 1625-1632, December 2013

25. Long, J., Shelhamer, E., Darrell, T.: Fully convolutional networks for semantic segmentation. In: IEEE Conference on Computer Vision and Pattern Recognition (CVPR) (2015)

26. Mottaghi, R., Chen, X., Liu, X., Cho, N.G., Lee, S.W., Fidler, S., Urtasun, R., Yuille, A.: The role of context for object detection and semantic segmentation in the wild. In: 2014 IEEE Conference on Computer Vision and Pattern Recognition, pp. 891-898 (2014)

27. Silberman, N., Hoiem, D., Kohli, P., Fergus, R.: Indoor segmentation and support inference from RGBD images. In: Fitzgibbon, A., Lazebnik, S., Perona, P., Sato, Y., Schmid, C. (eds.) ECCV 2012. LNCS, vol. 7578, pp. 746-760. Springer, Heidelberg (2012). doi:10.1007/978-3-642-33715-4_54

28. Gupta, S., Girshick, R., Arbeláez, P., Malik, J.: Learning rich features from RGBD images for object detection and segmentation. In: Fleet, D., Pajdla, T., Schiele, B., Tuytelaars, T. (eds.) ECCV 2014. LNCS, vol. 8693, pp. 345-360. Springer, Heidelberg (2014). doi:10.1007/978-3-319-10584-0_23 